\title{
Interplay of three G-quadruplex units in the KIT promoter
}

Cosimo Ducani, ${ }^{* 1}$ Giulio Bernardinelli, ${ }^{1}$ Björn Högberg, ${ }^{1}$ Bernhard K. Keppler, ${ }^{2}$ Alessio Terenzi, $*, 2$

1 Department of Medical Biochemistry and Biophysics, Karolinska Institutet, Stockholm, Sweden

${ }^{2}$ Institute of Inorganic Chemistry, University of Vienna, Waehringerstrasse 42, A-1090 Vienna, Austria

\section{Supporting Info}

Table S1. Short single stranded sequences used in this study.

Table S2. Long single stranded sequences used in this study.

Table S3. Addgene ID of the constructs.

Figure S1. CD spectra of the short wild-type and mutated sequences.

Figure S2. NUPACK analysis of short and long ODNs at $37^{\circ} \mathrm{C}$ (G-to-A mutations).

Figure S3. NUPACK analysis of short and long ODNs at $37^{\circ} \mathrm{C}$ (G-to-T mutations).

Figure S4. CD spectra of the long wild-type and mutated sequences.

Figure S5. CD spectra of the long wild-type and mutated oligos compared with the short oligos. p.S6

$\begin{array}{lr}\text { Figure S6. CD spectra at increasing temperature. } & \text { p.S7 }\end{array}$

Figure S7. ImageJ analysis of EMSA with long ODNs after staining with Thioflavin T. p.S8

Figure S8. Fluorescence at $488 \mathrm{~nm}$ upon addition of ThT to the indicated short and long ODNs. p.S8

Figure S9. Fluorescence titration of long ODNs with ThT. p.S9

Figure S10. Linearization of the 186 bp long dsDNA and crowding EMSA bands analysis. p.S9

Figure S11. EMSA negative controls of 186 bp long wild-type dsDNA p.S10

Figure S12. ImageJ analysis of the 186 bp long wild-type dsDNA p.S10 
Table S1. 5' $-3^{\prime}$ sequences of short single stranded sequences used in this study.

\begin{tabular}{|c|l|}
\hline WK2 & CGGGCGGGCGCGAGGGAGGGG \\
\hline MK2 & CAAACAAACGCGAAAAGAAA \\
\hline MK2 (G-to-T) & CTTTCTTTCGCGATTTAGTTT \\
\hline WSP & GGCGAGGAGGGGCGTGGCCGGC \\
\hline MSP & GACGAGAAGAAACGTGACCGAC \\
\hline MSP (G-to-T) & GTCGAGTAGTTTCGTGTCCGTC \\
\hline WK1 & AGGGAGGGCGCTGGGAGGAGGGG \\
\hline MK1 & AAAGAGAACGCTAAGAGGAGGAA \\
\hline MK1 (G-to-T) & ATTGAGTTCGCTTTGAGGAGGTT \\
\hline
\end{tabular}

Table S2. 5'-3' sequences of long single stranded sequences used in this study.

\begin{tabular}{|l|l|}
\hline WK2-WSP-WK1 & AGACCCGGGCGGCGCGAGGGAGGGGAGGCGAGGAGGGGCGTGGCCGGCGCGCAGAGGGAGGGCGCTGGGAGGAGGGG \\
\hline MK2-WSP-WK1 & AGACCCAAACAAACGCGAAAAAGAAAGGGAGGAGGGCGTGGCCGGCGGCAGAGGGAGGCGCTGGGGGAGGGG \\
\hline WK2-MSP-WK1 & AGACCCGGGCGGGCGCGAGGGAGGGGAGACGAGAAGAAACGTGACCGACGCGCAGAGGGAGGGCGCTGGGAGGAGGGG \\
\hline WK2-WSP-MK1 & AGACCCGGGCGGCGCGAGGGAGGGGAGGCGAGGAGGGGGTGGCCGGCGCGAGAAAGAGAACGCTAAGAGGAGGAA \\
\hline MK2-MSP-WK1 & AGACCCAAACAAACGCGAAAAAGAAAAGACGAGAAGAAACGTGACCGACGCGCAGAGGGAGGGCGCTGGGAGGAGGGG \\
\hline MK2-WSP-MK1 & AGACCCAAACAAACGCGAAAAAGAAAAGGCGAGGAGGGGCGTGGCCGGCGCGCAGAAAGAGAACGCTAAGAGGAGGAA \\
\hline WK2-MSP-MK1 & AGACCCGGGGGGCGCGAGGGAGGGGAGACGAGAAGAAACGTGACCGACGCGCAGAAAGAGAACGCTAAGAGGAGGAA \\
\hline MK2-MK1-MSP & AGACCCAAACAAACGCGAAAAAGAAAAGACGAGAAGAAACGTGACCGACGCGCAGAAAGAGAACGCTAAGAGGAGGAA \\
\hline
\end{tabular}

Table S3. Addgene identification number of the constructs containing KIT sequences with wild-type and mutated $\mathrm{G}$ units.

\begin{tabular}{|l|l|}
\hline Plasmid ID & Plasmid \\
\hline 118983 & pC-KIT1 \\
\hline 118984 & pC-KIT2 \\
\hline 118985 & pC-KIT3 \\
\hline 118986 & pC-KIT4 \\
\hline 118987 & pC-KIT5 \\
\hline 118988 & pC-KIT6 \\
\hline 118989 & pC-KIT7 \\
\hline 118990 & pC-KIT8 \\
\hline
\end{tabular}



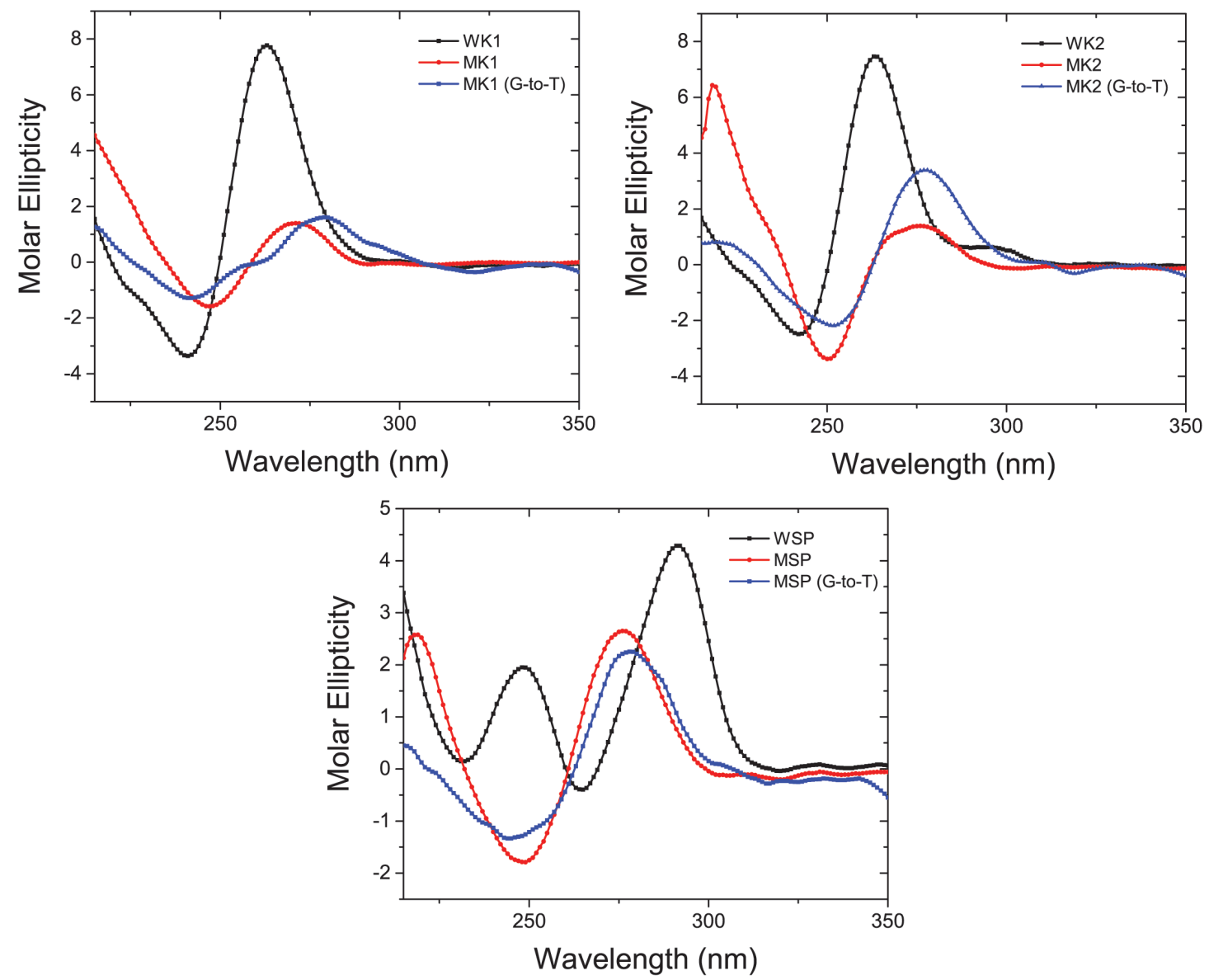

Figure S1. CD spectra of the short wild-type sequences (WK1, WK2 and WSP) in comparison with the spectra of the corresponding mutated versions (both G-to-A and G-to-T). Buffer: Tris-HCl $50 \mathrm{mM}(\mathrm{pH}=7.4)$ supplemented with $\mathrm{KCl} 100 \mathrm{mM}$. Units for molar ellipticity in CD spectra are $10^{6} \mathrm{mdeg}^{-1} \mathrm{~cm}^{-1}$. 

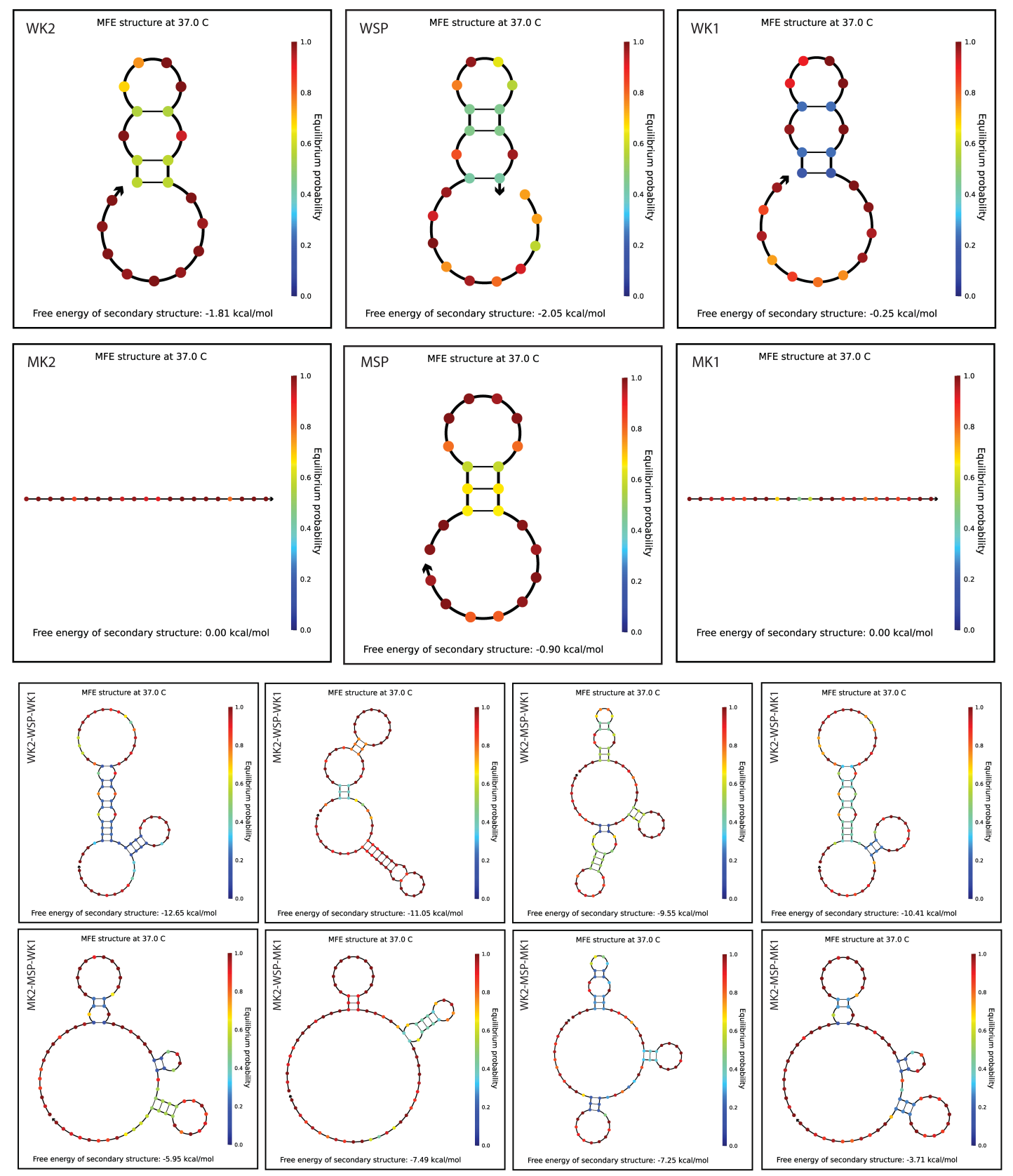

Figure S2. NUPACK analysis of short and long ODNs at $37^{\circ} \mathrm{C}$ : all the oligonucleotides, with the different combinations of wild-type and mutated (G-to-A) G4 units showing very similar free energy values and quite unstable secondary structures. 


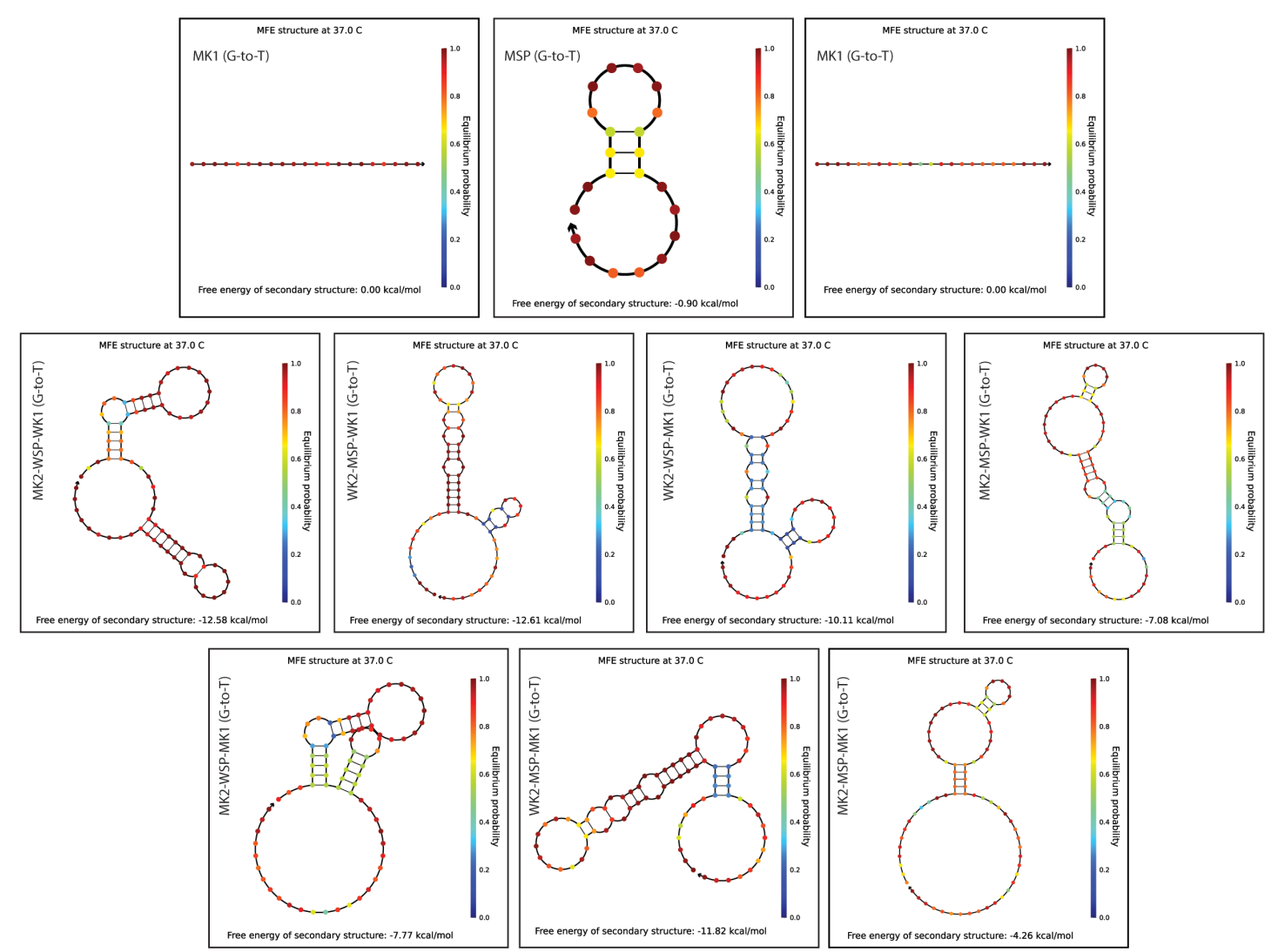

Figure S3. NUPACK analysis of short and long ODNs at $37^{\circ} \mathrm{C}$ : the oligonucleotides with the G-to-T mutated sequences.
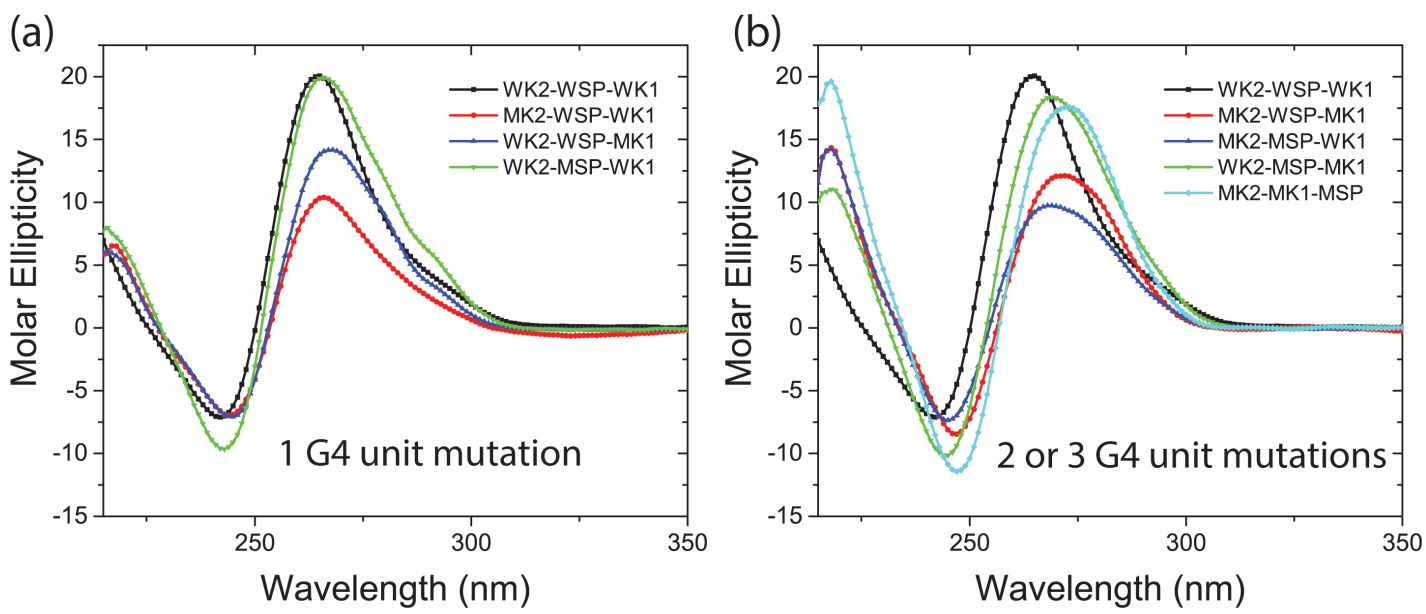

Figure S4. CD spectra of the long wild-type sequence WK2-WSP-WK1 in comparison with the spectra of (a) the corresponding sequences with mutations on one G4 unit and (b) the sequences with two or three mutated units. Buffer: Tris- $\mathrm{HCl} 50 \mathrm{mM}(\mathrm{pH}=$ 7.4) supplemented with $\mathrm{KCl} 100 \mathrm{mM}$. Units for molar ellipticity in $\mathrm{CD}$ spectra are $10^{6}$ mdeg $\mathrm{M}^{-1} \mathrm{~cm}^{-1}$. 
(a)

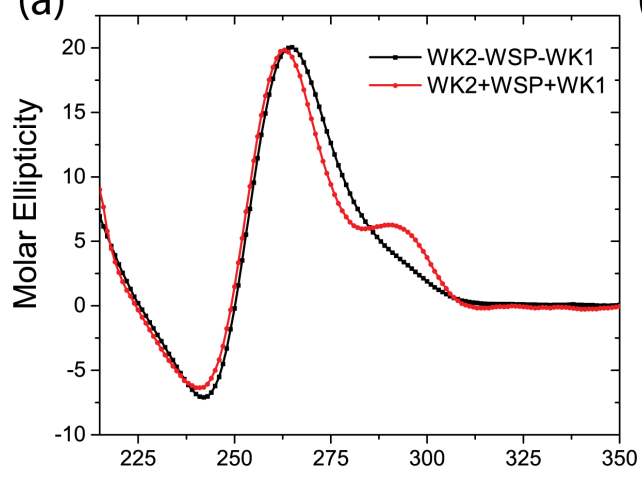

(c)

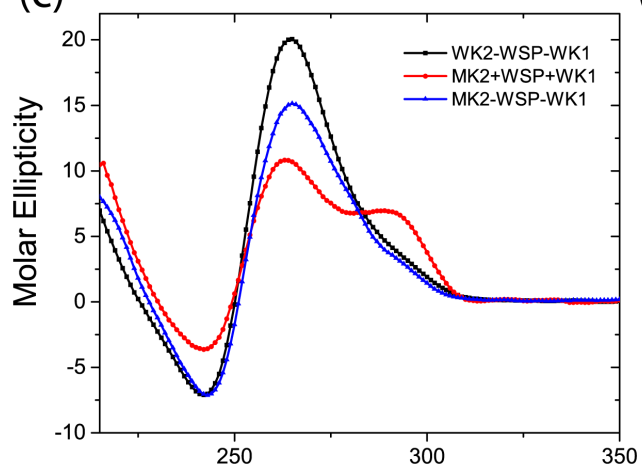

(e)

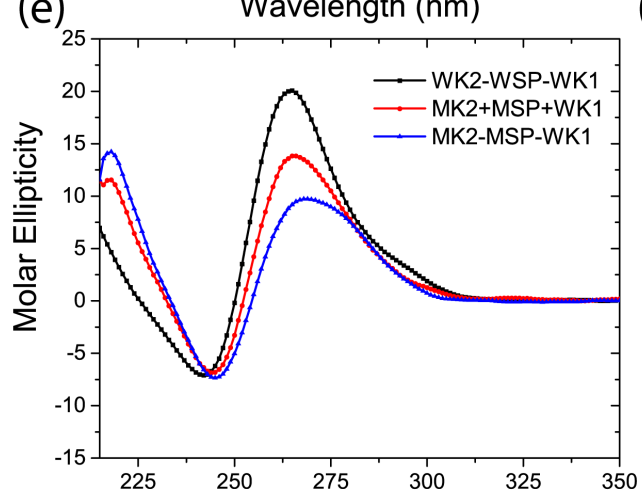

(g)

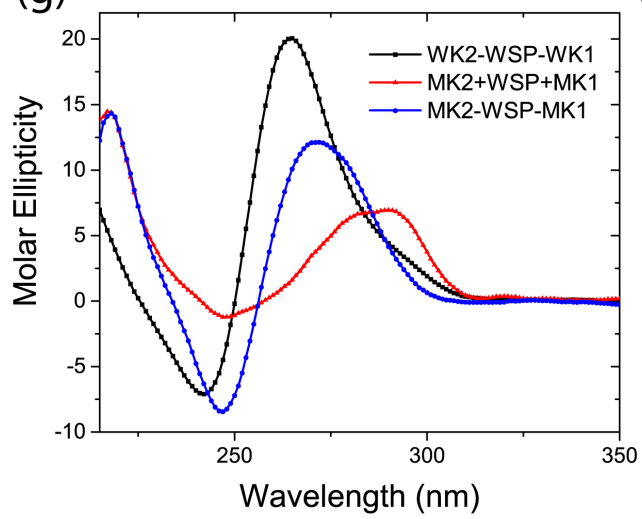

(b)

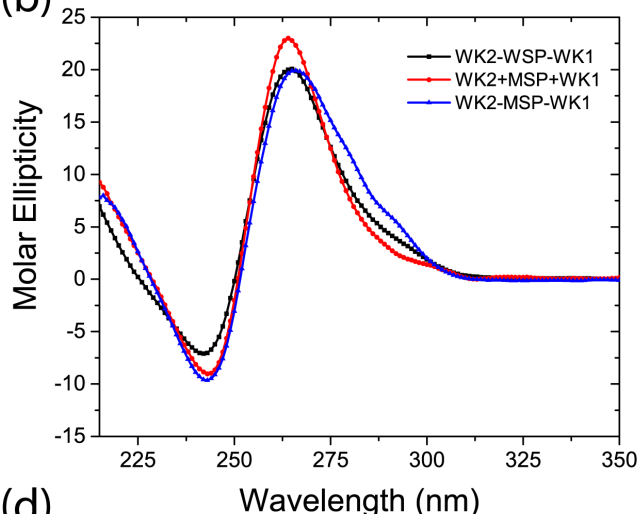

(d)

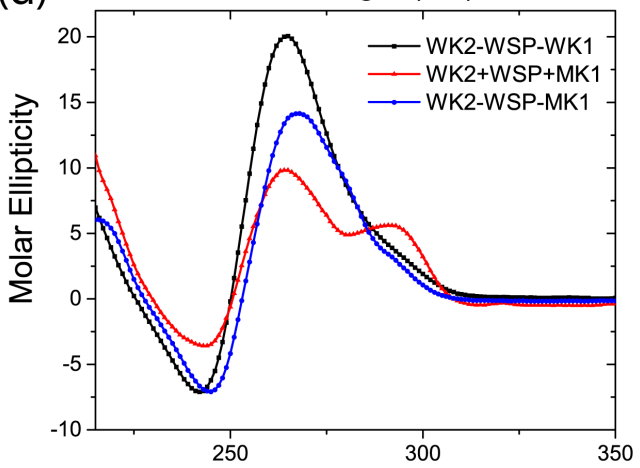

(f)

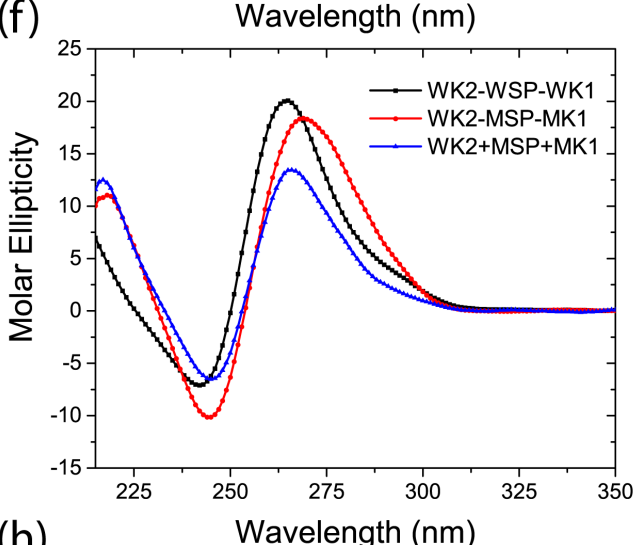

(h)

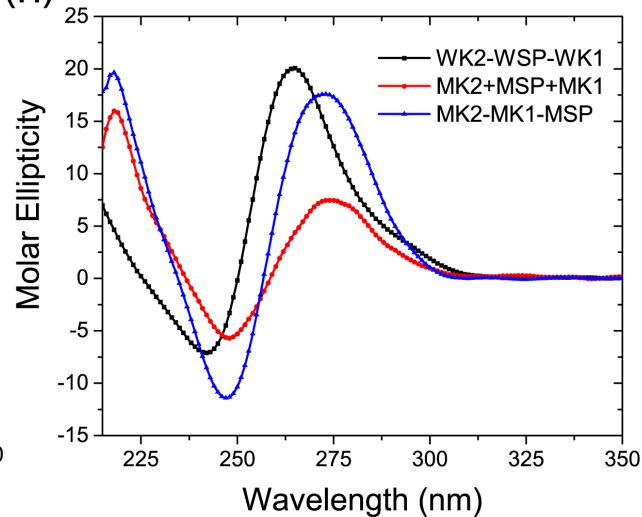

Figure S5. CD spectra of the long wild-type sequence WK2-WSP-WK1 (black lines) compared with the long sequences with mutations on one, two or three G4 units (blue lines) and the spectrum produced by the mixture of the corresponding pre-folded short sequences (either wild-type or mutated) (red lines). Buffer: Tris- $\mathrm{HCl} 50 \mathrm{mM}$ (pH = 7.4) supplemented with $\mathrm{KCl} 100 \mathrm{mM}$. Units for molar ellipticity in CD spectra are $10^{6}$ mdeg $\mathrm{M}^{-1} \mathrm{~cm}^{-1}$. 

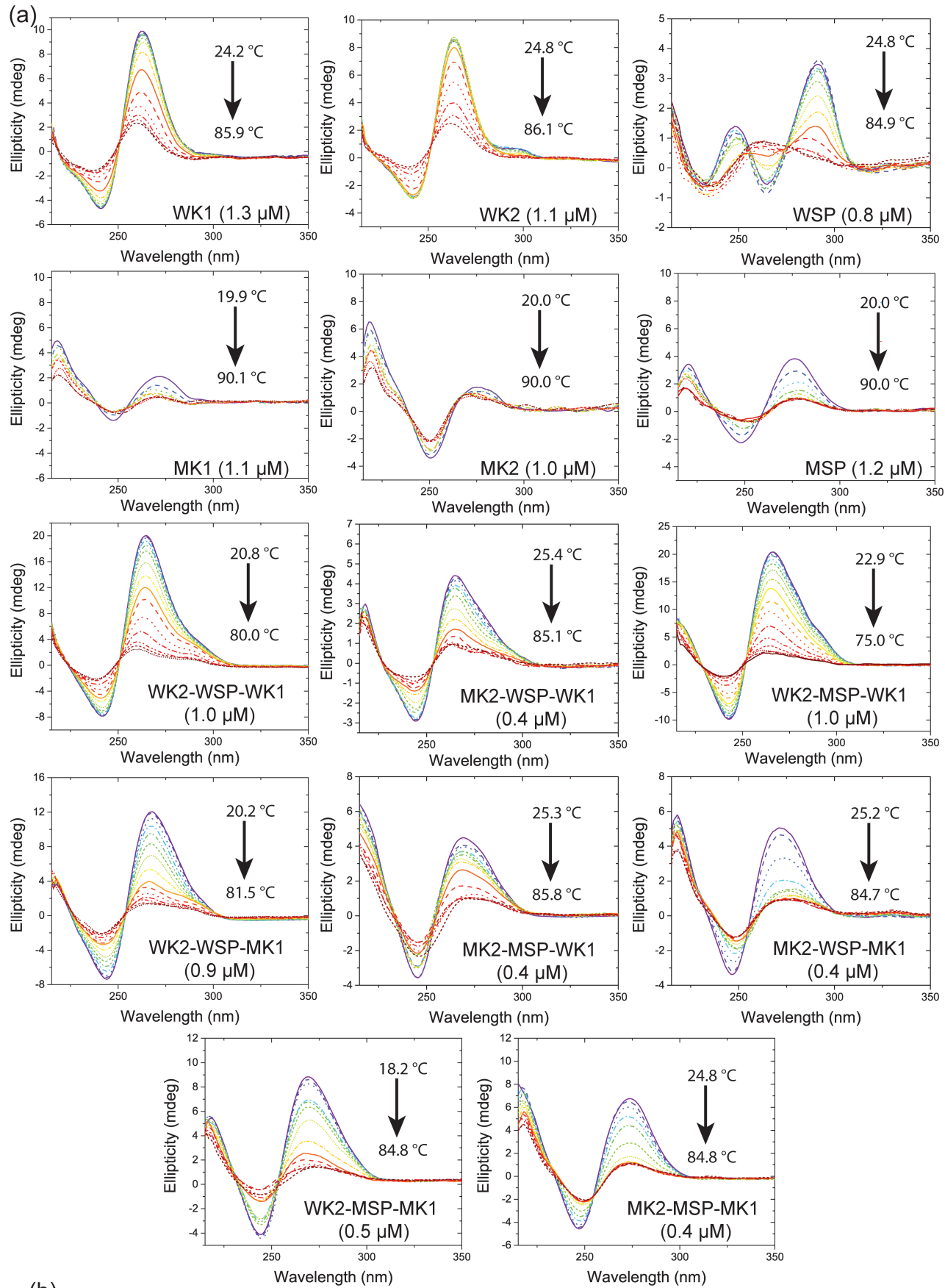

(b)
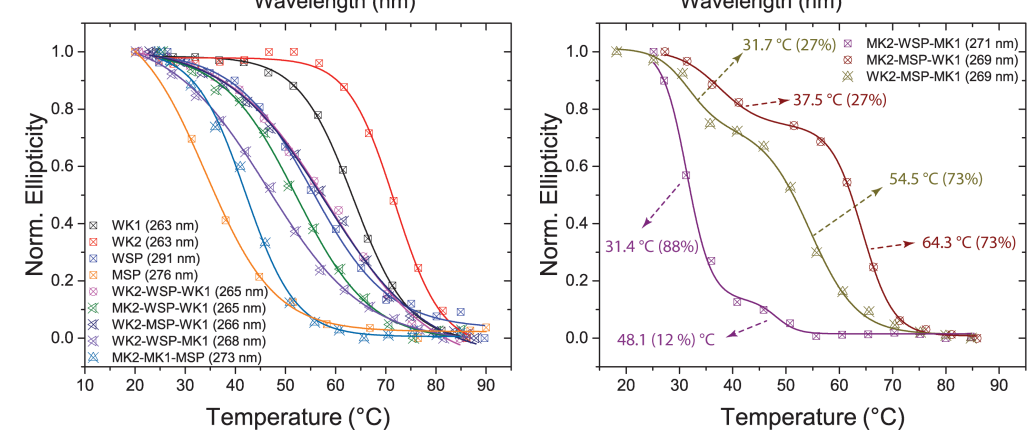

Figure S6. (a). CD spectra at increasing temperature of the indicated ODNs. Buffer: Tris- $\mathrm{HCl} 50 \mathrm{mM}(\mathrm{pH}=7.4)$ supplemented with $\mathrm{KCl} 100 \mathrm{mM}$. (b) Melting curves at the indicated wavelength and corresponding sigmoidal fits. For double mutated ODNs, analysis of the multiple transitions is reported. 


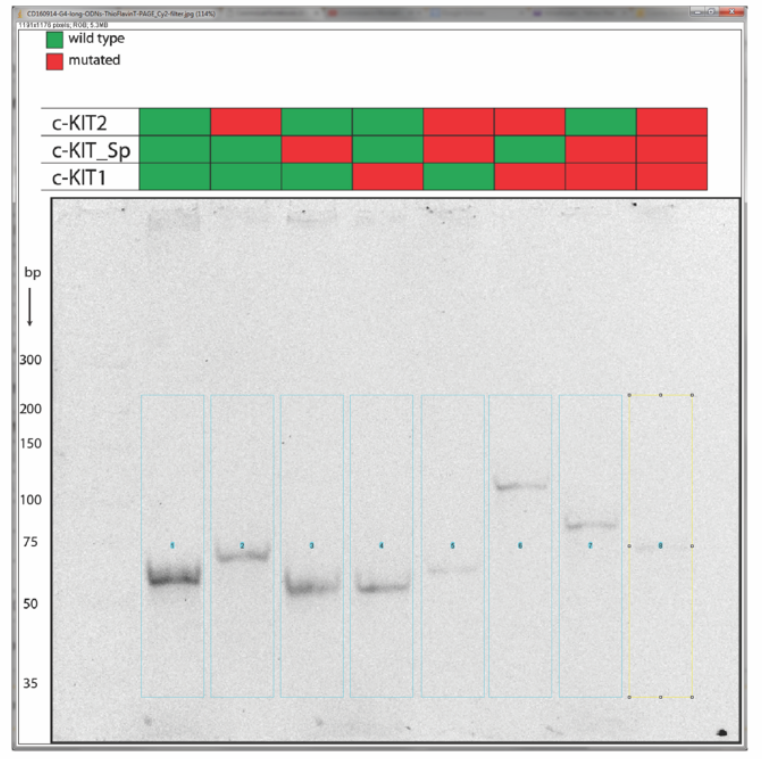

\begin{tabular}{|c|c|c|c|}
\hline ODN & Intensities & $\Delta$-Intensities & Ratios \\
\hline WK2-WSP-WK1 & 12005 & 11352 & 6.01 \\
\hline MK2-WSP-WK1 & 4538 & 3885 & 2.06 \\
\hline WK2-MSP-WK1 & 6853 & 6200 & 3.28 \\
\hline WK2-WSP-MK1 & 4329 & 3676 & 1.95 \\
\hline MK2-MSP-WK1 & 726 & 73 & 0,04 \\
\hline MK2-WSP-MK1 & 2586 & 1933 & 1.02 \\
\hline WK2-MSP-MK1 & 2540 & 1887 & 1 \\
\hline MK2-MSP-MK1 & 653 (*) $^{*}$ & 0 & 0 \\
\hline
\end{tabular}

${ }^{*}$ ) subtracted intensity to get $\Delta$-Intensities

Figure S7. ImageJ analysis of EMSA with long ODNs after staining with Thioflavin T (ThT): before calculating the intensity ratios, from each intensity value, we subtracted the intensity value of the fully mutated oligonucleotides (MK2-MSP-MK1), considered background fluorescence due to unspecific interaction of ThT with ssDNA.
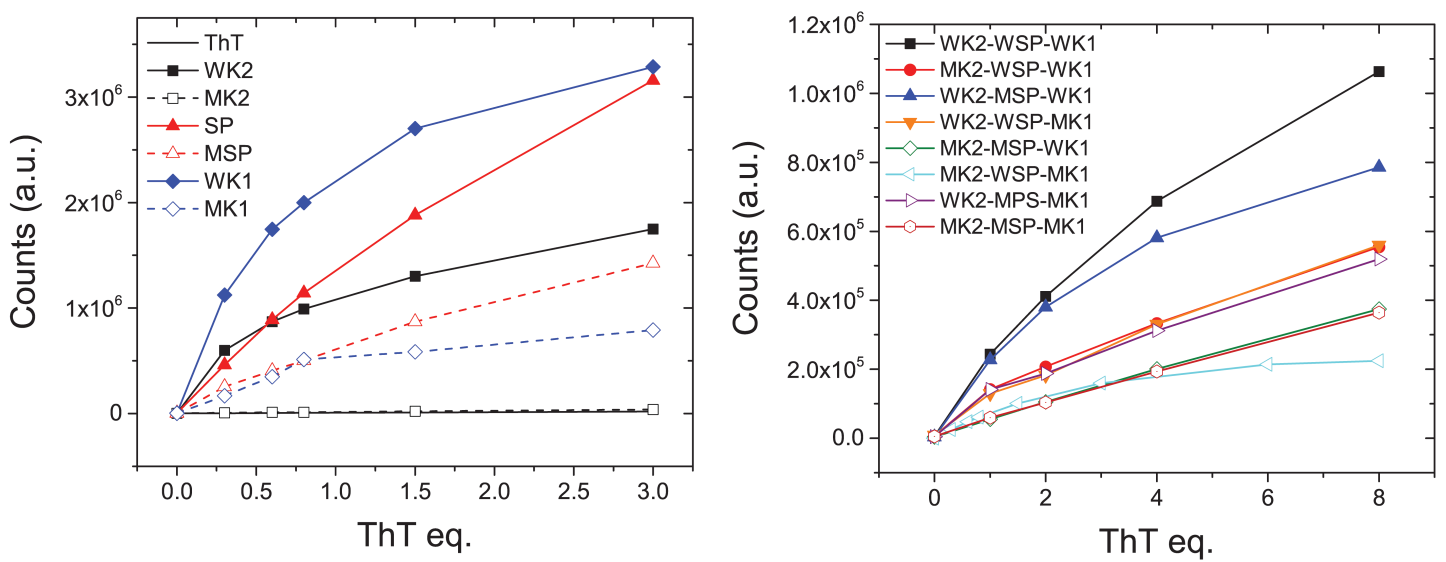

Figure S8. Fluorescence enhancement at $488 \mathrm{~nm}$ upon addition of ThT to the indicated short (left) and long (right) ODNs. $\lambda_{\text {exc }}=425 \mathrm{~nm}$, Tris-HCl $50 \mathrm{mM}$ buffer $(\mathrm{pH}=7.4)$ supplemented with $\mathrm{KCl} 100 \mathrm{mM}$. 

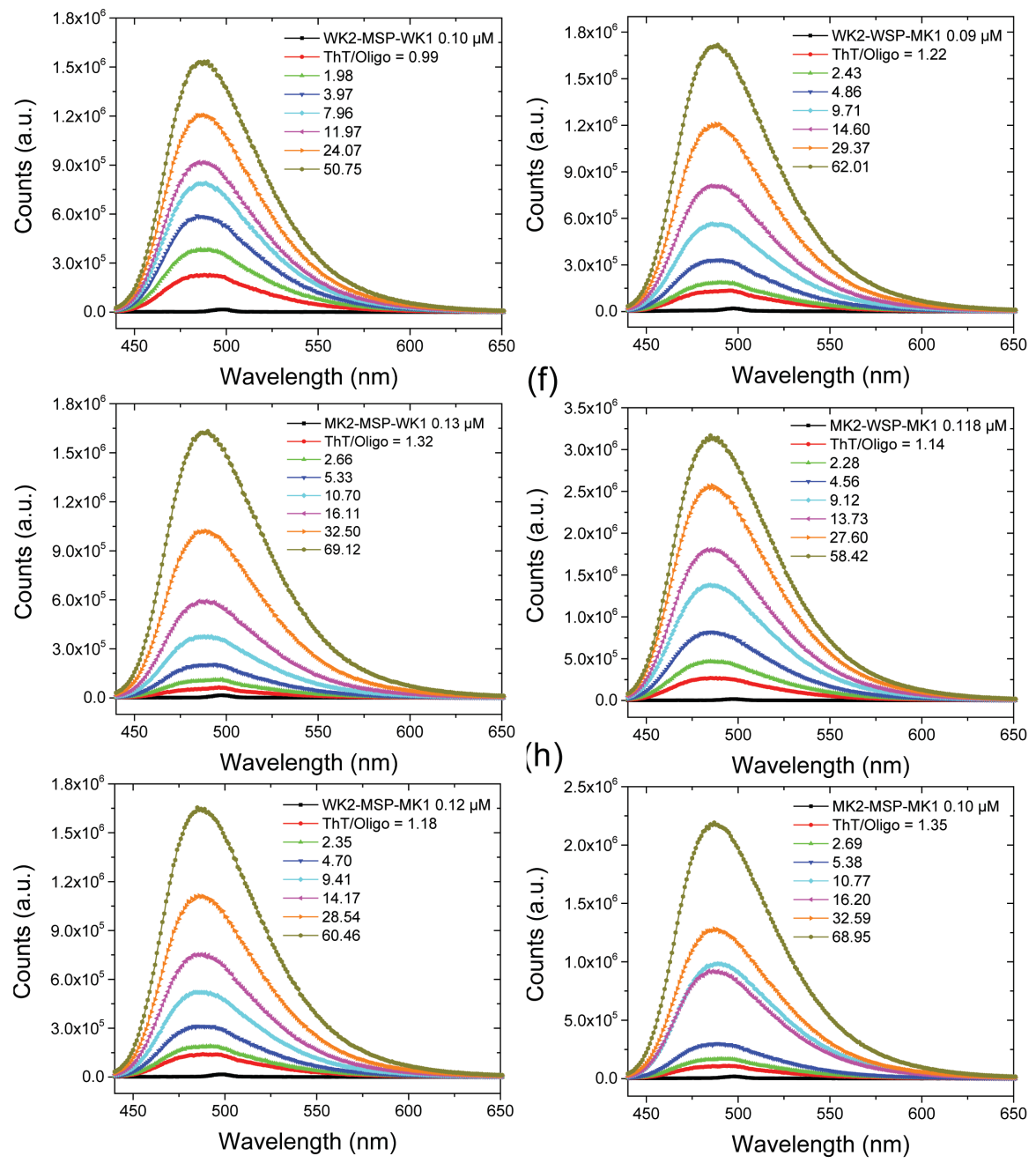

Figure 59. Fluorescence titration of long ODNs with ThT at the indicated ThT/ODN ratio. $\lambda_{\text {exc }}=425 \mathrm{~nm}$, Tris- $\mathrm{HCl} 50 \mathrm{mM}$ buffer $(\mathrm{pH}=7.4)$ supplemented with $\mathrm{KCl} 100 \mathrm{mM}$.

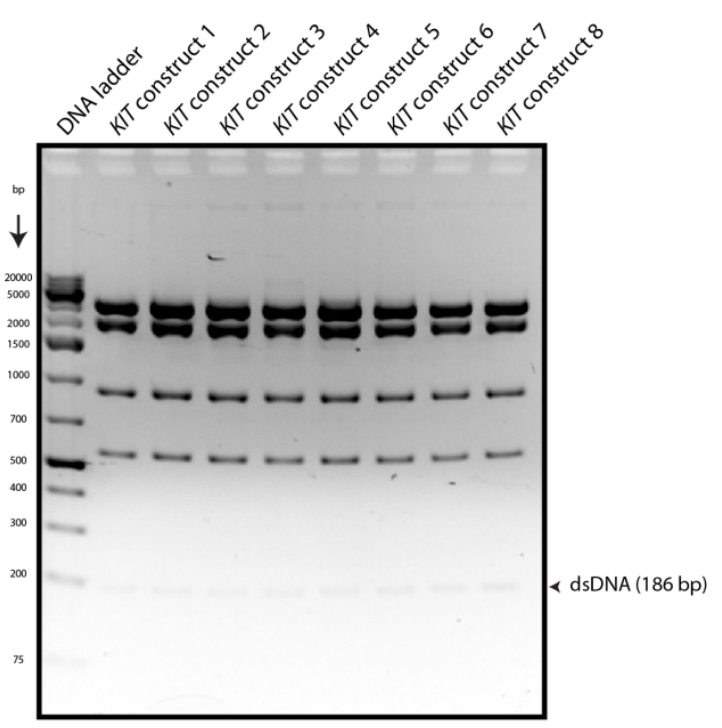

Figure S10. Linearization of the 186 bp long dsDNA containing the three $\mathrm{G} 4$ units in their wild-type and/or mutated version. 


\begin{tabular}{l|c|c|c|c|c|}
\hline Heating & + & + & + & + & - \\
\hline $\mathrm{KCl}$ & + & - & + & - & + \\
\hline PEG200 & + & - & - & + & + \\
\hline
\end{tabular}

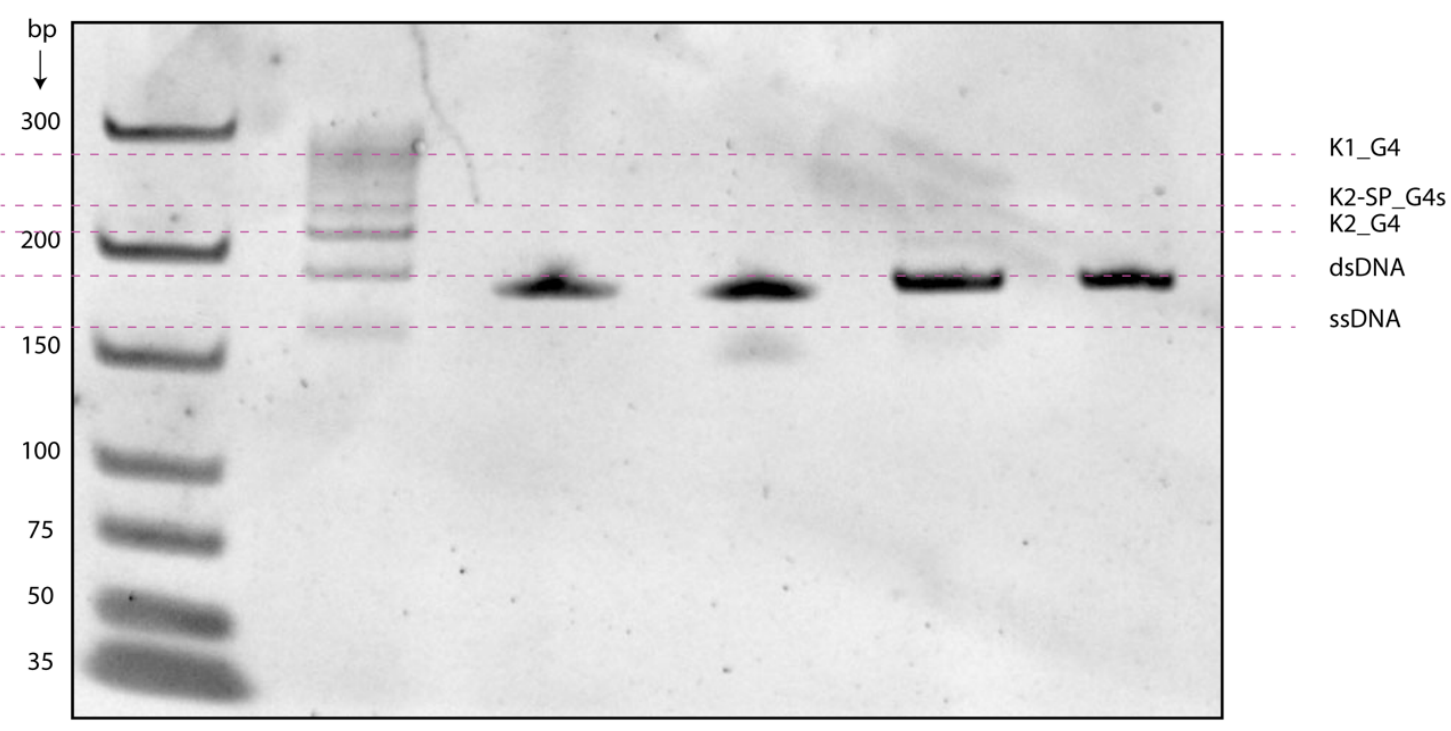

Figure S11. EMSA performed on dsDNA wild-type sequence in presence $(+)$ or absence (-) of heating (denaturation-renaturation step), $\mathrm{KCl}$ and PEG200.

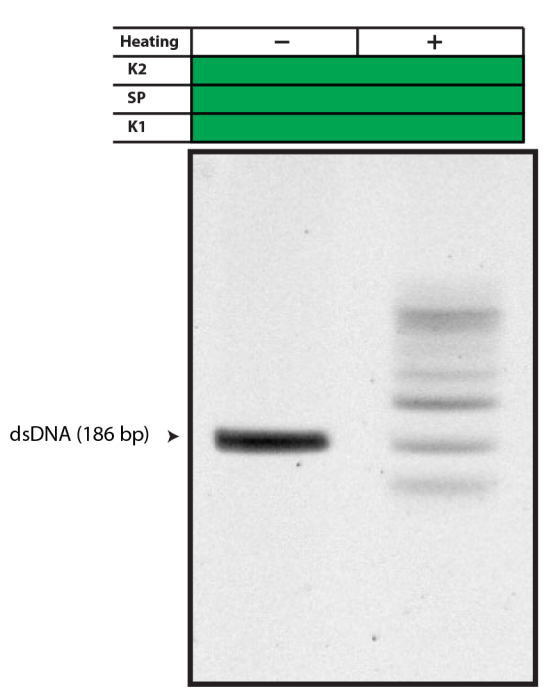

Wild type

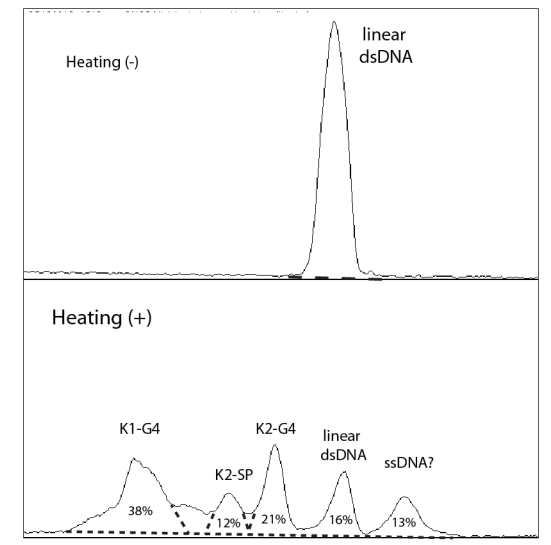

Figure S12. ImageJ analysis of the 186 bp dsDNA wild-type KIT core promoter in molecular crowding EMSA, before and after denaturation-renaturation steps (heating). The most represented product, after renaturation is represented by $\mathrm{G} 4$ formation of the K1 unit. 